\title{
Hemichannels in neurodegenerative diseases: is there a link to pathology?
}

\author{
Megan Bosch ${ }^{1}$ and Tammy Kielian ${ }^{2 *}$ \\ ' Department of Pharmacology and Experimental Neuroscience, University of Nebraska Medical Center, Omaha, NE, USA \\ ${ }^{2}$ Department of Pathology and Microbiology, University of Nebraska Medical Center, Omaha, NE, USA
}

\author{
Edited by: \\ Juan Andrés Orellana, Pontificia \\ Universidad Católica de Chile, Chile \\ Reviewed by: \\ Juan C. Saez, Universidad Catolica \\ de Chile, Chile \\ Christian Giaume, Collège de \\ France, France \\ *Correspondence: \\ Tammy Kielian, Department of \\ Pathology and Microbiology, \\ University of Nebraska Medical \\ Center, 985900 Nebraska Medical \\ Center, Omaha, \\ NE 68198-5900, USA \\ e-mail:tkielian@unmc.edu
}

Although originally considered a structural component of gap junctions, connexin hemichannels (HCs) are now recognized as functional entities capable of influencing metabolic gradients within the CNS, allowing direct communication between the intraand extracellular milieus. Besides connexins, HCs can also be formed by pannexins, which are not capable of gap junction assembly. Both positive and negative effects have been attributed to $\mathrm{HC}$ activity in the context of neurodegenerative diseases. For example, $\mathrm{HCs}$ can exert neuroprotective effects by promoting the uptake of neurotoxic molecules, whereas chronic $\mathrm{HC}$ opening can disrupt molecular gradients leading to cellular dysfunction and death. The latter scenario has been suggested for multiple neurodegenerative disorders, including Alzheimer's disease (AD) and more recently, lysosomal storage disorders, which are the focus of this perspective. Currently available evidence suggests a complex role for HCs in neurodegenerative disorders, which sets the stage for future studies to determine whether targeting $\mathrm{HC}$ action may improve disease outcomes.

Keywords: connexin, hemichannels, neurodegeneration, lysosomal storage disease, Alzheimer's disease

\section{INTRODUCTION}

Hemichannels (HCs) are composed of six connexin (Cx) subunits that assemble into hexameric pores that traffic to the plasma membrane where they can remain uncoupled or pair with adjacent HCs on neighboring cells to form gap junction channels (Contreras et al., 2003). Besides Cxs, HCs can also be formed by pannexins (Panx), which are not capable of gap junction assembly. This Perspective focuses on HC involvement in neurodegenerative diseases; therefore, gap junction activity will not be discussed here but has been the subject of several excellent reviews related to CNS disorders (Orellana et al., 2009; Eugenin et al., 2012). HCs are permeable to small hydrophilic molecules, such as ATP, $\mathrm{Ca}^{2+}$, glutamate, glucose, and glutathione, which are critical for CNS homeostasis by maintaining ionic and metabolic gradients and can also control autocrine/paracrine signaling (Retamal et al., 2007; Kielian, 2008; Rouach et al., 2008; Sánchez et al., 2009; Schalper et al., 2010; Orellana et al., 2011a; Bennett et al., 2012; Fiori et al., 2014).

Currently, a total of $10 \mathrm{Cx}$ and 2 Panx isoforms have been reported to be expressed in the brain (Giaume et al., 2013). The repertoire of $\mathrm{Cx}$ protein expression is distinct among various CNS cell types; however, the functional impact of these differences remains to be fully defined. For example, astrocytes primarily express $\mathrm{Cx} 43$ and $\mathrm{Cx} 30$, as well as, $\mathrm{Cx} 26, \mathrm{Cx} 40, \mathrm{Cx} 45$ and $\mathrm{Cx} 46$; microglia utilize $\mathrm{Cx} 43, \mathrm{Cx} 36$, and $\mathrm{Cx} 32$; and neurons express Cx36, Cx26, Cx45, and Cx57 (Rouach et al., 2002; Mika and Prochnow, 2012). Studies have shown that a selective pattern of $\mathrm{Cx} \mathrm{HC}$ expression may orchestrate extracellular signaling networks between various glial cell types and/or neurons in a homotypic or heterotypic fashion (Giaume et al., 2013; May et al., 2013). Panx expression has been reported in neurons, astrocytes, and more recently microglia (Orellana et al., 2009, 2011b; Sáez et al., 2013b); however, an outstanding question is whether $\mathrm{Cx} /$ Panx HC function differs within various CNS cell subpopulations. For example, it is becoming clear that astrocytes exhibit regional heterogeneity and it will be interesting to determine whether this is associated with differences in the molecular composition of $\mathrm{Cx} / \mathrm{Panx} \mathrm{HCs}$ and/or sensitivity to $\mathrm{HC}$ opening.

Originally considered a structural component of gap junctions, strong evidence has emerged to support a role for HCs in maintaining cellular and tissue homeostasis, by allowing cells to directly communicate with their surrounding microenvironment and relay signals via the release of molecules that activate extracellular receptors in an autocrine/paracrine manner (Orellana et al., 2009). In the last decade, several reports have linked inflammation and dysregulated $\mathrm{HC}$ activity in the CNS. The summation of this work suggests that HCs have a dual role in regulating molecular gradient homeostasis in the context of neurodegenerative diseases. On the one hand, transient HC activity has been suggested to be protective during normal physiologic states as well as acute insults or inflammation. For example, astrocyte HCs have been shown to promote glucose uptake, which is considered a second glucose-lactate pathway for neurons. In addition, HCs could release lactate, which could also be beneficial for neurons (Rouach et al., 2008; Giaume et al., 2013). Proinflammatory cytokines (i.e., IL-1 $\beta$ and TNF- $\alpha$ ) are known to activate glia and promote 
HC opening, which may serve as a means to propagate glial activation or neuron activity by the release of bioactive molecules acting in an autocrine/paracrine manner. Conversely, sustained HC opening during chronic neurodegenerative diseases may promote disease progression by perturbing metabolic gradients and the exaggerated release of toxic molecules to induce cell death (Figure 1).

\section{HC INVOLVEMENT IN NEURODEGENERATIVE DISEASES}

A common denominator linking neurodegenerative diseases and HC opening is neuroinflammation, characterized by microglial and astrocyte activation and secretion of inflammatory mediators. Microglia, the resident macrophages of the CNS parenchyma, phagocytose cellular debris and produce a wide array of proinflammatory molecules (Kettenmann et al., 2011; Lyman et al., 2014). These mediators, which include cytokines (i.e., IL- $\beta$, IL-6, and $\mathrm{TNF}-\alpha$ ), reactive oxygen and nitrogen species, glutamate, and neurotrophic factors enhance cell mobility, phagocytosis, and can be neuroprotective when carefully regulated (Kielian, 2008; Mika and Prochnow, 2012). Under physiological conditions, transient astrocyte activation plays a pivotal role in maintaining neurotransmitter levels at the tripartite synapse and metabolite trafficking. However, during neuroinflammation, chronic proinflammatory mediator release may cause dramatic and potentially detrimental alterations in the way microglia and astrocytes communicate and regulate homeostasis via $\mathrm{HC}$ opening (Figure 1). The end result is a milieu that can lead to cellular toxicity or dysfunction, which over time, can manifest as cognitive and/or motor decline depending on the CNS site affected (Finn et al., 2011; Orellana et al., 2011a; Xiong and Kielian, 2013).

\section{HC ACTIVITY IN LYSOSOMAL STORAGE DISEASES}

Lysosomal storage diseases (LSDs) encompass a large group of inherited metabolic disorders characterized by the accumulation of storage material within lysosomes. Collectively, LSDs afflict 1 out of every 6700 live births and approximately $75 \%$ of the LSDs currently identified impact CNS function (Meikle et al., 1999; Sands and Haskins, 2008). Although a relatively new area of investigation, recent studies have reported perturbed HC activity in two distinct LSDs, namely Juvenile Neuronal Ceroid Lipofuscinosis (JNCL) and Niemann-Pick type C (NPC).

JNCL is caused by a mutation in the CLN3 gene that most commonly spans exons 7-8 (Janes et al., 1996; Cotman et al., 2002; Drack et al., 2013). Brains of JNCL patients at autopsy as well as JNCL mouse models have shown that areas of activated microglia and astrocytes correlate with regions of neuron loss, along with elevated levels of IL- $1 \beta$ and ceramide, the latter representing a key lipid mediator involved in inflammation and apoptosis (Pontikis et al., 2004; Mencarelli and MartinezMartinez, 2013; Xiong and Kielian, 2013). A recent study from our laboratory using primary microglia from the CLN3 ${ }^{\Delta \operatorname{ex} 7 / 8}$ mouse model of JNCL demonstrated that when challenged with "danger signals" elevated in the brains of JNCL patients (i.e., ceramide and neuron lysate), CLN3 ${ }^{\Delta \operatorname{ex} 7 / 8}$ microglia released significantly more proinflammatory mediators compared to wild type cells, which remained largely non-responsive (Xiong and Kielian,

Membrane Level
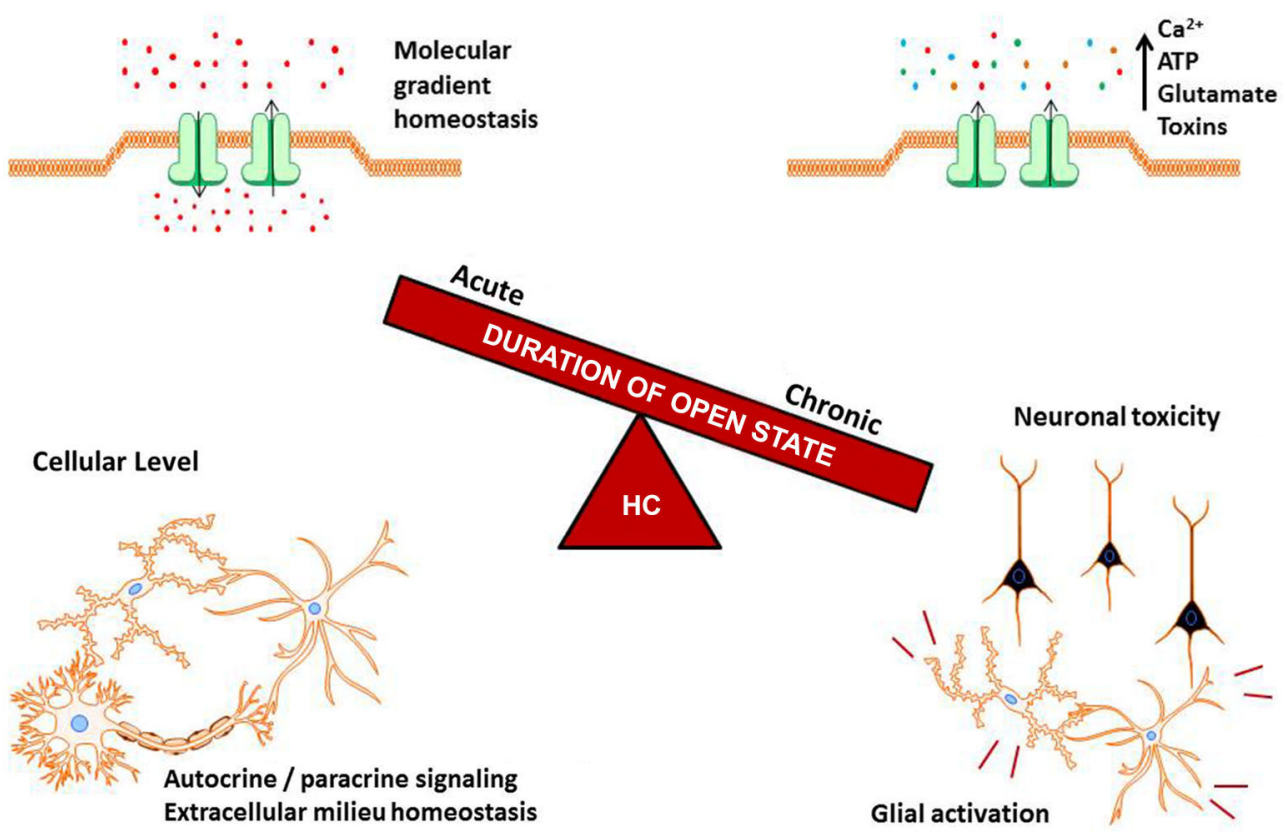

FIGURE 1 | Balance of hemichannel (HC) regulation in the CNS. HC opening is under tight control, where acute activity helps to maintain molecular gradients, cell homeostasis, and autocrine/paracine signaling. A slight disruption of this balance, as can occur during neurodegenerative diseases and inflammatory signals, causes increased $\mathrm{HC}$ opening. Chronic $\mathrm{HC}$ activity can result in the ablation of vital molecular gradients, toxin release into the extracellular milieu, and potentiation of glial activation and neuron death. 
2013). Furthermore, $\mathrm{CLN}^{\mathrm{Aex} 7 / 8}$ microglia displayed increased $\mathrm{HC}$ opening, which was associated with elevated glutamate and ATP release. Glutamate accumulation can cause neuronal excitotoxicity and a role for glutamate excitotoxicity in JNCL progression has been previously reported (Finn et al., 2011).

Another recent study from our laboratory revealed a transient increase in astrocyte $\mathrm{HC}$ activity in disease-affected regions of the $\mathrm{CLN}^{\Delta \mathrm{ex} 7 / 8}$ mouse brain as early as postnatal day 30 , which significantly preceded neuron loss that is not evident until 6-8 months of age (Burkovetskaya et al., 2014). However, this increase was transient, since CLN3 ${ }^{\Delta \mathrm{ex} 7 / 8}$ astrocyte HC function began to decline at postnatal day 60 , eventually falling below levels observed in wild type mice by postnatal day 90 , suggesting a progressive decline in astrocyte function at later stages of disease. Treatment of CLN3 ${ }^{\Delta \mathrm{ex} 7 / 8}$ mice with the HC inhibitor INI-0602, a blood-brain barrier permeable derivative of carbenoxolone (Takeuchi et al., 2011), significantly reduced lysosomal storage material accumulation in specific brain regions. In addition, astrocyte gap junction communication was significantly elevated in $\mathrm{CLN}^{\mathrm{Aex} 7 / 8}$ mice, which was predicted to occur via HC closure, although this was not apparent in acute brain slices ex vivo (Burkovetskaya et al., 2014). Nonetheless, aberrant HC activity in astrocytes and microglia may contribute to neuron loss in JNCL, particularly when considering that glial activation predates neuron death by several months in this mouse model (Pontikis et al., 2004). Unresolved questions are whether changes in HC function are responsible for the brain metabolic disturbances reported in JNCL and whether HC involvement extends to other CNS cell types (i.e., neurons and microglia).

NPC is caused by a mutation in the NPC1 or NPC2 genes, with the former being most common. NPC1 and NPC2 are required for cholesterol clearance and their absence causes the accumulation of cholesterol and other lipids in lysosomes (Rosenbaum and Maxfield, 2011). Similar to JNCL, neuroinflammation has been implicated in NPC pathology (Baudry et al., 2003). However, a recent study by Sáez et al. (2013a) suggested that the increase in HC activity observed in NPC might not be related to neuroinflammation per se but rather, the mutation itself (Sáez et al., 2013a). Specifically, primary astrocyte cultures from $\mathrm{NPC}^{-/-}$mice displayed increased $\mathrm{HC}$ activity under baseline conditions compared with cells from wild type and $\mathrm{NPC}^{+/-}$ animals. In addition, acute hippocampal slices from $\mathrm{NPC}^{-/-}$ mice at postnatal day 2 revealed increased astrocyte HC activity that could be blocked using the general $\mathrm{HC}$ blocker $\mathrm{La}^{3+}$ and $\mathrm{Cx} 43$ antibody. These data imply the involvement of $\mathrm{Cx} 43 \mathrm{HCs}$ and suggest that dysfunctional HCs occur at the earliest phase of NPC disease. It remains to be determined whether this HC activity represents an attempt by astrocytes to regain homeostasis in the context of NPC mutation or whether HC opening sets the stage for downstream neuropathology. Nevertheless, the available evidence supports a role for HCs in two distinct LSDs that have devastating consequences on the CNS (Finn et al., 2011; Sáez et al., 2013a; Burkovetskaya et al., 2014).

\section{ALZHEIMER'S DISEASE AND HC FUNCTION}

Alzheimer's disease (AD) is currently the leading cause of dementia in adults over the age of 65 years (Tiiman et al., 2013).
Hallmark symptoms of AD include memory impairment, loss of abstract thought and language skills, and alterations in personality (Welander et al., 2009). Pathologically, AD is characterized by the extracellular accumulation of amyloid-beta $(\mathrm{A} \beta)$ peptide into senile plaques and the intracellular hyperphosphorylation of tau protein into neurofibrillary tangles. These aggregates cause neuronal damage, generation of reactive oxygen and nitrogen species, neuroinflammation, and defects in cell-cell communication (Selkoe, 2001; Small and Duff, 2008; Quintanilla et al., 2012; von Bernhardi and Eugenin, 2012). The underlying mechanisms that elicit plaque and tangle accumulation in $\mathrm{AD}$ remain elusive.

Using an APP/PS1 mouse model of AD, Mei et al. (2010) observed increased $\mathrm{Cx} 43$ and $\mathrm{Cx} 30$ expression in astrocyte processes invading the plaque core and $\mathrm{Cx} 43$ immunoreactivity has also been associated with plaques in human AD tissues (Nagy et al., 1996; Mei et al., 2010). Along with increased Cx expression, $\mathrm{A} \beta$ has been reported to increase HC activity in neurons, astrocytes, and microglia (Orellana et al., 2011b). Similar to the series of events reported in JNCL above, $\mathrm{A} \beta$ elicits a proinflammatory response in resident glial cells typified by inflammatory cytokine, glutamate, and ATP release, which subsequently triggers HC opening in neighboring neurons. It is postulated that neuron $\mathrm{HC}$ opening is one factor responsible for neuron death that can further enhance neuroinflammation and propagate the neurodegenerative process.

Indeed, $\mathrm{HC}$ involvement in $\mathrm{AD}$ was also demonstrated by Takeuchi et al. (2011), where treatment of APP/PS1 mice with the HC inhibitor INI-0602 improved cognitive function (Takeuchi et al., 2011). In addition, INI-0602 blocked neurotoxic glutamate release from activated microglia both in vitro and in vivo. Although still in the relatively early stage of exploration, these findings suggest that targeting HCs could prove to be beneficial in combating $\mathrm{AD}$ symptoms and progression.

\section{CURRENT CHALLENGES IN THE FIELD OF HC BIOLOGY}

Since the discovery that HCs can exert functional activity, significant efforts have been made to characterize their roles in neurodegenerative diseases; however, many limitations still exist. One primary issue is the availability of reagents that can specifically block HC action. Many of the pharmacological inhibitors currently used to study HCs also affect gap junctions, which must be considered when these compounds are used experimentally. In addition, many widely used inhibitors are not selective for a particular $\mathrm{HC}$ type, which leaves the identification of $\mathrm{HC}$ protein composition in question. A potential solution is the use of $\mathrm{Cx}$-specific antibodies or $\mathrm{Cx} / \mathrm{Panx}$ mimetic peptides that can selectively block HC permeability (Sáez et al., 2013a). A second difficulty when studying $\mathrm{Cx} \mathrm{HCs}$ is the methods used to evaluate their activity, since it is challenging to distinguish between $\mathrm{Cx}$ gap junction and $\mathrm{HC}$ action. For example, HCs are often reported by increases in either $\mathrm{Cx}$ immunoreactivity in brain tissues or Western blots. However, both gap junctions and HCs are comprised of Cxs making it problematic to discriminate between the two. Functionally, the primary method used to measure HC activity is ethidium uptake. However, based on its low molecular weight, numerous types of open channels are permeable to ethidium, which calls to caution how HC ethidium uptake data 
is interpreted. Here the combination of ethidium uptake coupled with a $\mathrm{Cx} / \mathrm{Panx}$ selective inhibitor can begin to narrow the action to $\mathrm{Cx} /$ Panx channels; however, for the reasons mentioned above, identification of HC vs. gap junction channels remains an area of debate. Perhaps the best means to quantifying $\mathrm{HC}$ activity is single channel electrophysiology. This approach is feasible in cultured cells; however, it is significantly more challenging in brain slices, as reflected in a study by Kang et al. (2008) where electrophysiological evidence of HC activity was obtained in only 18 out of 700 recordings (Kang et al., 2008). Regardless of these technical limitations, careful consideration should be given to select the best model system to examine HC function. Many studies characterizing the functional roles of HCs have been performed in reconstituted liposomes or cell cultures (Fiori et al., 2014). While these methods provide useful insights, they are not able to recapitulate in vivo environments. Live tissue slices are better representative of in vivo events and have provided more functional data; however, this approach also has its limitations. Specifically, upon excision, the brain slice begins to slowly deteriorate due to cell damage inflicted during the cutting process concomitant with microglial activation, which may impact the results obtained. To ensure tissue viability for an extended period of time, slices are continuously bathed with artificial cerebrospinal fluid which is equilibrated with $\mathrm{CO}_{2}$ and care should be taken to evaluate cells that are positioned well below the cut surface to avoid signals from damaged regions. Future research avenues could be directed towards discovering new minimally invasive methods for studying HCs in vivo such as two-photon microscopy or channel tracers that can be imaged using functional magnetic resonance imaging (MRI) or single photon emission computed topography (SPECT; Kielian, 2008).

\section{OUTSTANDING QUESTIONS AND CONCLUSIONS}

Significant progress has been made towards elucidating the functional role of HCs in neurodegenerative diseases; however, many unanswered questions remain. The first is whether HC dysfunction is an active contributor to disease pathology or merely a consequence. In the case of $\mathrm{AD}$, neuroinflammation indirectly increases $\mathrm{HC}$ channel activity in multiple cell types (Orellana et al., 2011a; Quintanilla et al., 2012). Numerous reports by others also support a similar relationship where HC activation and neuroinflammation act as co-factors, where one event influences the progression of the other. If correct, then therapeutically targeting HCs would only potentially slow disease progression but not be curative. In contrast, a direct connection between HC activity and inflammation does not appear to exist in NPC. Specifically, increased HC activity in neurons, astrocytes, and microglia was not induced by proinflammatory stimulators, suggesting that $\mathrm{HC}$ action precedes neuroinflammation in NPC. A similar case for changes in HC activity predating neuroinflammation is seen in JNCL, since HC dysfunction was observed early, whereas overt inflammation is not evident until later stages of disease (Xiong and Kielian, 2013; Burkovetskaya et al., 2014). The similarities between these two LSDs suggest that a core response may be operative that is not tied to inflammation per se. Nonetheless, as the intensity of the inflammatory response increases with advancing disease, it is likely that inflammatory mediators will impact $\mathrm{HC}$ activity, perhaps in a fashion that has already been described in $\mathrm{AD}$ and glial cell culture models (Figure 1). The complexity of HC composition combined with distinct expression patterns on various CNS cell types suggest that new insights are bound to emerge to account for this diversity in HCs in terms of CNS homeostasis and pathology.

\section{ACKNOWLEDGMENTS}

This work was supported by the National Institutes of Health National Institute of Neurological Disorders and Stroke (NINDS) R21NS084392-01A1, Bee For Battens, The Saoirse Foundation, and the UNMC Dean's Pediatric Research Fund (to Tammy Kielian).

\section{REFERENCES}

Baudry, M., Yao, Y., Simmons, D., Liu, J., and Bi, X. (2003). Postnatal development of inflammation in a murine model of Niemann-Pick type $\mathrm{C}$ disease: immunohistochemical observations of microglia and astroglia. Exp. Neurol. 184, 887903. doi: 10.1016/s0014-4886(03)00345-5

Bennett, M. V., Garre, J. M., Orellana, J. A., Bukauskas, F. F., Nedergaard, M., and Saez, J. C. (2012). Connexin and pannexin hemichannels in inflammatory responses of glia and neurons. Brain Res. 1487, 3-15. doi: 10.1016/j.brainres. 2012.08.042

Burkovetskaya, M., Karpuk, N., Xiong, J., Bosch, M., Boska, M. D., Takeuchi, H., et al. (2014). Evidence for Aberrant astrocyte hemichannel activity in Juvenile Neuronal Ceroid Lipofuscinosis (JNCL). PLoS One 9:e95023. doi: 10. 1371/journal.pone.0095023

Contreras, J. E., Saez, J. C., Bukauskas, F. F., and Bennett, M. V. (2003). Gating and regulation of connexin 43 (Cx43) hemichannels. Proc. Natl. Acad. Sci. U S A 100, 11388-11393. doi: 10.1073/pnas.1434298100

Cotman, S. L., Vrbanac, V., Lebel, L. A., Lee, R. L., Johnson, K. A., Donahue, L. R., et al. (2002). Cln3(Deltaex7/8) knock-in mice with the common JNCL mutation exhibit progressive neurologic disease that begins before birth. Hum. Mol. Genet. 11, 2709-2721. doi: 10.1093/hmg/11.22.2709

Drack, A. V., Miller, J. N., and Pearce, D. A. (2013). A novel c.1135_1138delCTGT mutation in CLN3 leads to juvenile neuronal ceroid lipofuscinosis. J. Child Neurol. 28, 1112-1116. doi: 10.1177/0883073813494812

Eugenin, E. A., Basilio, D., Saez, J. C., Orellana, J. A., Raine, C. S., Bukauskas, F., et al. (2012). The role of gap junction channels during physiologic and pathologic conditions of the human central nervous system. J. Neuroimmune Pharmacol. 7, 499-518. doi: 10.1007/s11481-012-9352-5

Finn, R., Kovacs, A. D., and Pearce, D. A. (2011). Altered sensitivity of cerebellar granule cells to glutamate receptor overactivation in the $\mathrm{Cln} 3$ (Deltaex7/8)knock-in mouse model of juvenile neuronal ceroid lipofuscinosis. Neurochem. Int. 58, 648-655. doi: 10.1016/j.neuint.2011.02.003

Fiori, M. C., Reuss, L., Cuello, L. G., and Altenberg, G. A. (2014). Functional analysis and regulation of purified connexin hemichannels. Front. Physiol. 5:71. doi: 10.3389/fphys.2014.00071

Giaume, C., Leybaert, L., Naus, C. C., and Saez, J. C. (2013). Connexin and pannexin hemichannels in brain glial cells: properties, pharmacology and roles. Front. Pharmacol. 4:88. doi: 10.3389/fphar.2013.00088

Janes, R. W., Munroe, P. B., Mitchison, H. M., Gardiner, R. M., Mole, S. E., and Wallace, B. A. (1996). A model for Batten disease protein CLN3: functional implications from homology and mutations. FEBS Lett. 399, 75-77. doi: 10. 1016/s0014-5793(96)01290-2

Kang, J., Kang, N., Lovatt, D., Torres, A., Zhao, Z., Lin, J., et al. (2008). Connexin 43 hemichannels are permeable to ATP. J. Neurosci. 28, 4702-4711. doi: 10. 1523/JNEUROSCI.5048-07.2008

Kettenmann, H., Hanisch, U. K., Noda, M., and Verkhratsky, A. (2011). Physiology of microglia. Physiol. Rev. 91, 461-553. doi: 10.1152/physrev.00011.2010

Kielian, T. (2008). Glial connexins and gap junctions in CNS inflammation and disease. J. Neurochem. 106, 1000-1016. doi: 10.1111/j.1471-4159.2008.05405.X

Lyman, M., Lloyd, D. G., Ji, X., Vizcaychipi, M. P., and Ma, D. (2014). Neuroinflammation: the role and consequences. Neurosci. Res. 79, 1-12. doi: 10.1016/j. neures.2013.10.004 
May, D., Tress, O., Seifert, G., and Willecke, K. (2013). Connexin47 protein phosphorylation and stability in oligodendrocytes depend on expression of Connexin43 protein in astrocytes. J. Neurosci. 33, 7985-7996. doi: 10. 1523/JNEUROSCI.5874-12.2013

Mei, X., Ezan, P., Giaume, C., and Koulakoff, A. (2010). Astroglial connexin immunoreactivity is specifically altered at beta-amyloid plaques in $\beta$-amyloid precursor protein/presenilin1 mice. Neuroscience 171, 92-105. doi: 10.1016/j. neuroscience.2010.08.001

Meikle, P. J., Hopwood, J. J., Clague, A. E., and Carey, W. F. (1999). Prevalence of lysosomal storage disorders. JAMA 281, 249-254. doi: 10.1001/jama.281.3.249

Mencarelli, C., and Martinez-Martinez, P. (2013). Ceramide function in the brain: when a slight tilt is enough. Cell. Mol. Life Sci. 70, 181-203. doi: 10.1007/s00018012-1038-x

Mika, T., and Prochnow, N. (2012). Functions of connexins and large pore channels on microglial cells: the gates to environment. Brain Res. 1487, 16-24. doi: 10. 1016/j.brainres.2012.07.020

Nagy, J. I., Li, W., Hertzberg, E. L., and Marotta, C. A. (1996). Elevated connexin43 immunoreactivity at sites of amyloid plaques in Alzheimer's disease. Brain Res. 717, 173-178. doi: 10.1016/0006-8993(95)01526-4

Orellana, J. A., Froger, N., Ezan, P., Jiang, J. X., Bennett, M. V., Naus, C. C., et al. (2011a). ATP and glutamate released via astroglial connexin 43 hemichannels mediate neuronal death through activation of pannexin 1 hemichannels. $J$. Neurochem. 118, 826-840. doi: 10.1111/j.1471-4159.2011.07210.x

Orellana, J. A., Saez, P. J., Shoji, K. F., Schalper, K. A., Palacios-Prado, N., Velarde, V., et al. (2009). Modulation of brain hemichannels and gap junction channels by pro-inflammatory agents and their possible role in neurodegeneration. Antioxid. Redox Signal. 11, 369-399. doi: 10.1089/ars.2008.2130

Orellana, J. A., Shoji, K. F., Abudara, V., Ezan, P., Amigou, E., Saez, P. J., et al. (2011b). Amyloid $\beta$-induced death in neurons involves glial and neuronal hemichannels. J. Neurosci. 31, 4962-4977. doi: 10.1523/JNEUROSCI.6417-10. 2011

Pontikis, C. C., Cella, C. V., Parihar, N., Lim, M. J., Chakrabarti, S., Mitchison, H. M., et al. (2004). Late onset neurodegeneration in the Cln3-/- mouse model of juvenile neuronal ceroid lipofuscinosis is preceded by low level glial activation. Brain Res. 1023, 231-242. doi: 10.1016/j.brainres.2004.07.030

Quintanilla, R. A., Orellana, J. A., and von Bernhardi, R. (2012). Understanding risk factors for Alzheimer's disease: interplay of neuroinflammation, connexinbased communication and oxidative stress. Arch. Med. Res. 43, 632-644. doi: 10. 1016/j.arcmed.2012.10.016

Retamal, M. A., Froger, N., Palacios-Prado, N., Ezan, P., Saez, P. J., Saez, J. C., et al. (2007). Cx43 hemichannels and gap junction channels in astrocytes are regulated oppositely by proinflammatory cytokines released from activated microglia. J. Neurosci. 27, 13781-13792. doi: 10.1523/jneurosci.2042-07.2007

Rosenbaum, A. I., and Maxfield, F. R. (2011). Niemann-Pick type C disease: molecular mechanisms and potential therapeutic approaches. J. Neurochem. 116, 789-795. doi: 10.1111/j.1471-4159.2010.06976.x

Rouach, N., Avignone, E., Meme, W., Koulakoff, A., Venance, L., Blomstrand, F., et al. (2002). Gap junctions and connexin expression in the normal and pathological central nervous system. Biol. Cell. 94, 457-475. doi: 10.1016/s02484900(02)00016-3

Rouach, N., Koulakoff, A., Abudara, V., Willecke, K., and Giaume, C. (2008). Astroglial metabolic networks sustain hippocampal synaptic transmission. Science 322, 1551-1555. doi: 10.1126/science.1164022

Sáez, P. J., Orellana, J. A., Vega-Riveros, N., Figueroa, V. A., Hernandez, D. E., Castro, J. F., et al. (2013a). Disruption in connexin-based communication is associated with intracellular $\mathrm{Ca}(2)(+)$ signal alterations in astrocytes from Niemann-Pick type C mice. PLoS One 8:e71361. doi: 10.1371/journal.pone. 0071361

Sáez, P. J., Shoji, K. F., Retamal, M. A., Harcha, P. A., Ramirez, G., Jiang, J. X., et al. (2013b). ATP is required and advances cytokine-induced gap junction formation in microglia in vitro. Mediators Inflamm. 2013:216402. doi: 10 . $1155 / 2013 / 216402$

Sánchez, H. A., Orellana, J. A., Verselis, V. K., and Saez, J. C. (2009). Metabolic inhibition increases activity of connexin-32 hemichannels permeable to Ca2+ in transfected HeLa cells. Am. J. Physiol. Cell Physiol. 297, C665-C678. doi: 10. 1152/ajpcell.00200.2009

Sands, M. S., and Haskins, M. E. (2008). CNS-directed gene therapy for lysosomal storage diseases. Acta Paediatr. Suppl. 97, 22-27. doi: 10.1111/j.1651-2227.2008. 00660.x

Schalper, K. A., Sanchez, H. A., Lee, S. C., Altenberg, G. A., Nathanson, M. H., and Saez, J. C. (2010). Connexin 43 hemichannels mediate the Ca2+ influx induced by extracellular alkalinization. Am. J. Physiol. Cell Physiol. 299, C1504-C1515. doi: 10.1152/ajpcell.00015.2010

Selkoe, D. J. (2001). Alzheimer's disease results from the cerebral accumulation and cytotoxicity of amyloid beta-protein. J. Alzheimers Dis. 3, 75-80.

Small, S. A., and Duff, K. (2008). Linking Abeta and tau in late-onset Alzheimer's disease: a dual pathway hypothesis. Neuron $60,534-542$. doi: 10.1016/j.neuron. 2008.11.007

Takeuchi, H., Mizoguchi, H., Doi, Y., Jin, S., Noda, M., Liang, J., et al. (2011). Blockade of gap junction hemichannel suppresses disease progression in mouse models of amyotrophic lateral sclerosis and Alzheimer's disease. PLoS One 6:e21108. doi: 10.1371/journal.pone.0021108

Tiiman, A., Palumaa, P., and Tougu, V. (2013). The missing link in the amyloid cascade of Alzheimer's disease-metal ions. Neurochem. Int. 62, 367-378. doi: 10. 1016/j.neuint.2013.01.023

von Bernhardi, R., and Eugenin, J. (2012). Alzheimer's disease: redox dysregulation as a common denominator for diverse pathogenic mechanisms. Antioxid. Redox Signal. 16, 974-1031. doi: 10.1089/ars.2011.4082

Welander, H., Franberg, J., Graff, C., Sundstrom, E., Winblad, B., and Tjernberg, L. O. (2009). Abeta43 is more frequent than Abeta40 in amyloid plaque cores from Alzheimer disease brains. J. Neurochem. 110, 697-706. doi: 10.1111/j.14714159.2009.06170.x

Xiong, J., and Kielian, T. (2013). Microglia in juvenile neuronal ceroid lipofuscinosis are primed toward a pro-inflammatory phenotype. J. Neurochem. 127, 245-258. doi: 10.1111/jnc. 12385

Conflict of Interest Statement: The authors declare that the research was conducted in the absence of any commercial or financial relationships that could be construed as a potential conflict of interest.

Received: 30 June 2014; accepted: 31 July 2014; published online: 20 August 2014. Citation: Bosch M and Kielian T (2014) Hemichannels in neurodegenerative diseases: is there a link to pathology? Front. Cell. Neurosci. 8:242. doi: 10.3389/fncel.2014.00242 This article was submitted to the journal Frontiers in Cellular Neuroscience.

Copyright (C) 2014 Bosch and Kielian. This is an open-access article distributed under the terms of the Creative Commons Attribution License (CC BY). The use, distribution or reproduction in other forums is permitted, provided the original author(s) or licensor are credited and that the original publication in this journal is cited, in accordance with accepted academic practice. No use, distribution or reproduction is permitted which does not comply with these terms. 